(from http://www.healthfromnature.com/alternative. html): "The research contained on this site is general in nature and has been compiled, reviewed and corrected to the best of the authors ability. It is presented for educational purposes. It is your personal responsibility to determine the validity of any and all information presented. Each nutritional supplement product is NOT a drug and NOT represented as having any medicinal value." Presumably, the authors feel that this warning is sufficient to ward off any legal trouble should any misinformation provided lead to harm.

Readers interested in more information on unorthodox and unconventional clinical therapies can obtain a comprehensive critique of these methods at http://www.quackwatch.com and http://www. hcrc.org. The latter resource contains a rich selection of links to anti-quackery sites.

The power of the Internet is like a two-edged sword. It can deliver misinformation and uncorroborated opinion with equal ease. The thoughtful and the thoughtless co-exist side by side in cyberspace.

D. John Doyle MD PHD FRCPC

Toronto, Ontario

\section{ROMs and Anesthesia Machines}

At a recent conference I was given two free CD ROMs by the kind man at the Datex-Ohmeda booth. The first, bearing the pedestrian title "AS/3 Anesthesia Delivery Unit", uses Macromedia Director to provide an interactive multimedia presentation about their anesthesia machine. Although the CD ROM is very nicely done, it is still basically just a fancy marketing brochure, being well illustrated, and well narrated, but very short on technical detail. One advantage of providing marketing information in this way is that the per copy cost of producing a CD ROM can be much less than producing complex multicolour brochures that we associate with the marketing of high-technology medical products. Another advantage is that CD ROMs usually takes up less storage space. Incidentally, Datex-Ohmeda also use CD ROM technology for distributing all the technical manuals for their products. This means that it is easy to search electronically for obscure information and it even would be possible to provide video clips showing how correct disassembly and reassembly should be carried out.

The second CD ROM I received is much, much more than a marketing "throw away" and is entitled "The Evolution of the Anesthesia Machine". You will definitely want keep your copy of this CD ROM. This instructional product was conceived and developed by Drs. Ortega and Arkoff, both practitioners at Boston University at the time. It is a marvelous multimedia historical survey of the evolution of the technology used in our profession, and is again produced using Macromedia Director. This project was carried out in collaboration with the Wood Library Museum of the American Society of Anesthesiologists and was awarded first prize for being the outstanding scientific and educational exhibit at ASA annual meeting in 1998. Next month I will provide a complete review of this outstanding product. Meanwhile, if the history of our profession interests you, do try to get your own copy.

\author{
D. John Doyle MD PHD FRCPC \\ Toronto, Ontario
}

